\title{
The impact of long-term azithromycin on antibiotic resistance in HIV-associated chronic lung disease
}

\author{
Regina E. Abotsi $\mathbb{1}^{1,2}$, Mark P. Nicol ${ }^{3}$, Grace McHugh ${ }^{4}$, Victoria Simms $\mathbb{D}^{4,5}$, Andrea M. Rehman $\mathbb{D}^{5}$, \\ Charmaine Barthus $^{6}$, Lucky G. Ngwira ${ }^{7,8}$, Brenda Kwambana-Adams ${ }^{9}$, Robert S. Heyderman ${ }^{9}$, \\ Jon $\varnothing$. Odland ${ }^{10,11,12}$, Rashida A. Ferrand ${ }^{4,13}$, Felix S. Dube ${ }^{1}$ and The BREATHE study team
}

\begin{abstract}
${ }^{1}$ Department of Molecular and Cell Biology \& Institute of Infectious Diseases and Molecular Medicine, University of Cape Town, Cape Town, South Africa. ${ }^{2}$ Department of Pharmaceutical Microbiology, School of Pharmacy, University of Health and Allied Sciences, Ho, Ghana. ${ }^{3}$ Division of Infection and Immunity, School of Biomedical Sciences, Faculty of Health and Medical Sciences, University of Western Australia, Perth, Australia. ${ }^{4}$ Biomedical Research and Training Institute, Harare, Zimbabwe. ${ }^{5}$ International Statistics and Epidemiology Group, London School of Hygiene and Tropical Medicine, London, United Kingdom. ${ }^{6}$ Division of Medical Microbiology, University of Cape Town, Cape Town, South Africa. ${ }^{7}$ Malawi-Liverpool Wellcome Trust Clinical Research Programme, Blantyre, Malawi. ${ }^{8}$ Liverpool School of Tropical Medicine, Liverpool, United Kingdom. ${ }^{9}$ NIHR Global Health Research Unit on Mucosal Pathogens, Research Department of Infection, Division of Infection and Immunity, University College London, London, United Kingdom. ${ }^{10}$ Department of Community Medicine, University of Tromsø, Tromsø, Norway. ${ }^{11}$ International Research Laboratory for Reproductive Ecotoxicology (IL RET), The National Research University Higher School of Economics, Moscow, Russia. ${ }^{12}$ Faculty of Health Sciences, School of Health Systems and Public Health, University of Pretoria, Pretoria, South Africa. ${ }^{13}$ Clinical Research Department, London School of Hygiene and Tropical Medicine, London, United Kingdom.
\end{abstract}

Corresponding author: Regina E. Abotsi (abtreg001@myuct.ac.za, rekabotsi@gmail.com)

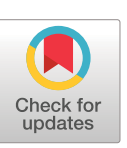

Copyright @The authors 2022

This version is distributed under the terms of the Creative Commons Attribution Non-Commercial Licence 4.0. For commercial reproduction rights and permissions contact permissions@ersnet.org

Received: 5 July 2021 Accepted: 31 Oct 2021

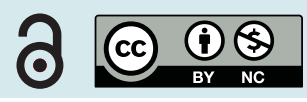

\section{Shareable abstract (@ERSpublications)}

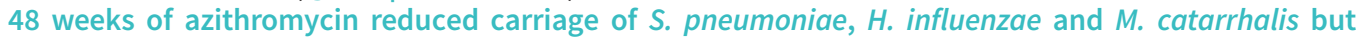
promoted antibiotic resistance in S. pneumoniae and S. aureus; antibiotic-resistant S. aureus persisted at 72 weeks from randomisation https://bit.ly/3LOm5tU

Cite this article as: Abotsi RE, Nicol MP, McHugh G, et al. The impact of long-term azithromycin on antibiotic resistance in HIV-associated chronic lung disease. ERJ Open Res 2022; 8: 00491-2021 [DOI: 10.1183/23120541.00491-2021].

\section{Abstract}

Selection for resistance to azithromycin (AZM) and other antibiotics such as tetracyclines and lincosamides remains a concern with long-term AZM use for treatment of chronic lung diseases (CLD). We investigated the impact of 48 weeks of AZM on the carriage and antibiotic resistance of common respiratory bacteria among children with HIV-associated CLD.

Nasopharyngeal (NP) swabs and sputa were collected at baseline, 48 and 72 weeks from participants with HIV-associated CLD randomised to receive weekly AZM or placebo for 48 weeks and followed postintervention until 72 weeks. The primary outcomes were prevalence and antibiotic resistance of Streptococcus pneumoniae (SP), Staphylococcus aureus (SA), Haemophilus influenzae (HI) and Moraxella catarrhalis (MC) at these timepoints. Mixed-effects logistic regression and Fisher's exact test were used to compare carriage and resistance, respectively.

Of 347 (174 AZM, 173 placebo) participants (median age 15 years (IQR 13-18), female 49\%), NP carriage was significantly lower in the AZM $(n=159)$ compared to placebo $(n=153)$ arm for SP $(18 \%$ versus $41 \%, p<0.001$ ), HI ( $7 \%$ versus $16 \%, \mathrm{p}=0.01$ ) and MC ( $4 \%$ versus $11 \%, \mathrm{p}=0.02$ ); SP resistance to AZM (62\% (18 out of 29 ) versus $13 \%$ (8 out of 63 ), $p<0.0001$ ) or tetracycline (60\% (18 out of 29) versus 21\% (13 out of 63), $p<0.0001$ ) was higher in the AZM arm. Carriage of SA resistant to AZM (91\% (31 out of 34) versus $3 \%$ (1 out of 31), $p<0.0001$ ), tetracycline (35\% (12 out of 34 ) versus $13 \%$ (4 out of 31), $\mathrm{p}=0.05$ ) and clindamycin (79\% (27 out of 34$)$ versus $3 \%$ ( 1 out of 31$), p<0.0001$ ) was also significantly higher in the AZM arm and persisted at 72 weeks. Similar findings were observed for sputa.

The persistence of antibiotic resistance and its clinical relevance for future infectious episodes requiring treatment needs further investigation.

Introduction

Long-term azithromycin (AZM) therapy is commonly used to manage chronic lung diseases (CLDs), including cystic fibrosis [1], non-cystic bronchiectasis [2], COPD [3] and asthma [4]. Evidence suggests 
that AZM may improve lung function and survival, and reduce frequency of acute pulmonary exacerbations, antibiotic administration and hospitalisation in some of these CLDs. These beneficial effects may be mediated by the dual antimicrobial and immunomodulatory properties of AZM [3].

However, AZM use can promote antibiotic resistance in both commensal and potentially pathogenic bacteria in the respiratory tract [1-4], which can be further spread to untreated individuals. AZM can also promote resistance to other antibiotics including tetracyclines, lincosamides and streptogramins [5]. This is mediated by alteration of drug targets shared by other antibiotics (lincosamides and streptogramins) and co-location of resistance genes on the same mobile genetic elements (tetracyclines) [5]. In a meta-analysis of CLD patients (COPD, cystic fibrosis, bronchiectasis and asthma) who received long-term AZM, the risk of bacterial resistance increased 2.7-fold, while bacterial colonisation risk was halved in the AZM group compared to placebo [6]. None of the trials included in this meta-analysis reported on the persistence of the microbiological effect post-intervention and none included African children or patients with HIV-related CLD. Two later studies that did investigate the persistence of the AZM's microbiological effect post-intervention could not provide robust conclusions due to few participants [2, 7] (mean of 25 per trial arm), short follow-up period (4 weeks for one study) [7] and variable timepoints for post-intervention samples [2].

Children with HIV are at high risk of respiratory tract infections. Recent studies have shown that these children also have a high prevalence of CLD, despite antiretroviral therapy (ART) [8]. The clinical presentation is typically that of chronic cough, reduced exercise tolerance, hypoxia and reduced lung function [8]. Up to a third of these children with HIV included in African studies have CLD with constrictive obliterative bronchiolitis being the dominant cause [8]. As there are currently no treatment guidelines, we conducted a double-blinded, placebo-controlled randomised trial that showed that 48 weeks of once-weekly AZM reduced acute respiratory exacerbations and all-cause hospitalisations in African children with HIV-associated CLD [9]. The microbiological sequelae of long-term AZM have not been previously investigated in a paediatric population with HIV-associated CLD. To address the potential for selection of antibiotic-resistant bacteria in this patient population, we investigated the impact of long-term AZM therapy on the carriage and antibiotic resistance of Streptococcus pneumoniae (SP), Staphylococcus aureus (SA), Haemophilus influenzae (HI) and Moraxella catarrhalis (MC) at 48 weeks (end of therapy) and 72 weeks from randomisation. We also assessed factors associated with carriage and resistance.

\section{Methods}

The main results of the BREATHE trial (ClinicalTrials.gov Identifier: NCT02426112) [9] are reported elsewhere. Briefly, individuals with HIV-associated CLD aged 6-19 years and taking ART for at least 6 months were enrolled from HIV outpatient clinics in Harare, Zimbabwe and Blantyre, Malawi. CLD was defined as forced expiratory volume in $1 \mathrm{~s}\left(\mathrm{FEV}_{1}\right) \mathrm{z}$-score $<-1.0$ with no reversibility $(<12 \%$ improvement in $\mathrm{FEV}_{1}$ after $200 \mu \mathrm{g}$ of salbutamol inhaled using a spacer). Participants were individually randomised to receive weight-based dosing of oral AZM (10 to $19.9 \mathrm{~kg}, 250 \mathrm{mg} ; 20$ to $29.9 \mathrm{~kg}, 500 \mathrm{mg}$; 30 to $39.9 \mathrm{~kg}$, $750 \mathrm{mg}$; and $40 \mathrm{~kg}$ or more, $1250 \mathrm{mg}$ ) or placebo once-weekly up to 48 weeks, with follow-up for a further 24 weeks post-intervention. Adherence to trial medication was defined as not missing, on average, more than two of the 12 dispensed doses, as assessed by pill count, splitting time in the study into four 12-week periods, according to visit and study medication dispensing schedule [9].

Nasopharyngeal (NP) swabs and sputa were collected from participants at baseline, 48- and 72-week visits. Details of sample collection, handling and transport, culture and antibiotic susceptibility testing (AST) for SP, SA, HI and MC have previously been described [10]. Briefly, susceptibility to AZM, tetracycline and cotrimoxazole was conducted for all four bacterial species using the Kirby-Bauer disk diffusion method. Additionally, AST was done for SP to oxacillin, SA to clindamycin, cefoxitin and penicillin, HI to amoxicillin-clavulanate, cefuroxime and ampicillin, and $\mathrm{MC}$ to amoxicillin-clavulanate. Inducible clindamycin resistance was assessed as previously reported [11]. SA susceptibility to cefoxitin was tested as a surrogate for methicillin resistance. AST was conducted in accordance with the 2018 Clinical and Laboratory Standards Institute guidelines and breakpoints [12].

\section{Outcomes}

The primary outcomes for this study were the difference in the prevalence of SP, SA, HI and MC and their AZM-resistant strains between trial arms at AZM cessation (48 weeks). Secondary outcomes included the differences in the carriage and AZM resistance between trial arms at 72 weeks. We also investigated the effect of long-term AZM on resistance to the additional antibiotics tested at both 48 and 72 weeks. The clinical and socio-demographic factors associated with the carriage of bacteria and AZM-resistant isolates 
at 48 and 72 weeks, and the effect of adherence to AZM on carriage and resistance (in the AZM arm only) were also assessed.

\section{Statistical analyses}

Statistical analyses were conducted in R software version 3.6.2. Bacterial carriage and antibiotic resistance were reported as percentages of total samples cultured and tested for resistance for each trial arm. Fisher's exact test and Mann-Whitney test were used to compare proportions and continuous variables, respectively. Comparisons of bacterial carriage between trial arms at all visits were conducted using mixed-effects logistic regression models, including a random effect for participants, and reported as adjusted odds ratios with 95\% confidence intervals in Stata version 15 (StataCorp, College Station, TX, USA). Models were adjusted for site, sex, age category, HIV viral load at baseline, the season of sampling and visit. A trial arm by time interaction term was included in models to compare trial arms at 48 and 72 weeks, without a trial arm main effect term [13].

\section{Ethical approval and consent to participate}

Approval for the main trial was obtained from local regulatory bodies at the study sites and the research ethics committees of the London School of Hygiene and Tropical Medicine, the University of Cape Town and the Medical and Health Research in Norway. This sub-study was approved by the Human Research Ethics Committee of the University of Cape Town (HREC/REF: 092/2019). Written informed consent and age-appropriate assent were provided by guardians and participants under 18 years, respectively. Older participants ( $\geqslant 18$ years) consented independently.

Results

Between June 15, 2016, and September 4, 2018, 347 participants (173 AZM and 174 placebo arm) were enrolled and randomised to AZM or placebo (figure 1). Eleven participants who were later found to not meet the inclusion criteria for $\mathrm{FEV}_{1}$ were included in this report. At baseline, participants had a median age of 15 years (IQR 13-18) with slightly more males than females (51\% (177 out of 347) versus 49\% (170 out of 347)). The majority of participants were on long-term cotrimoxazole prophylaxis (90\% (313

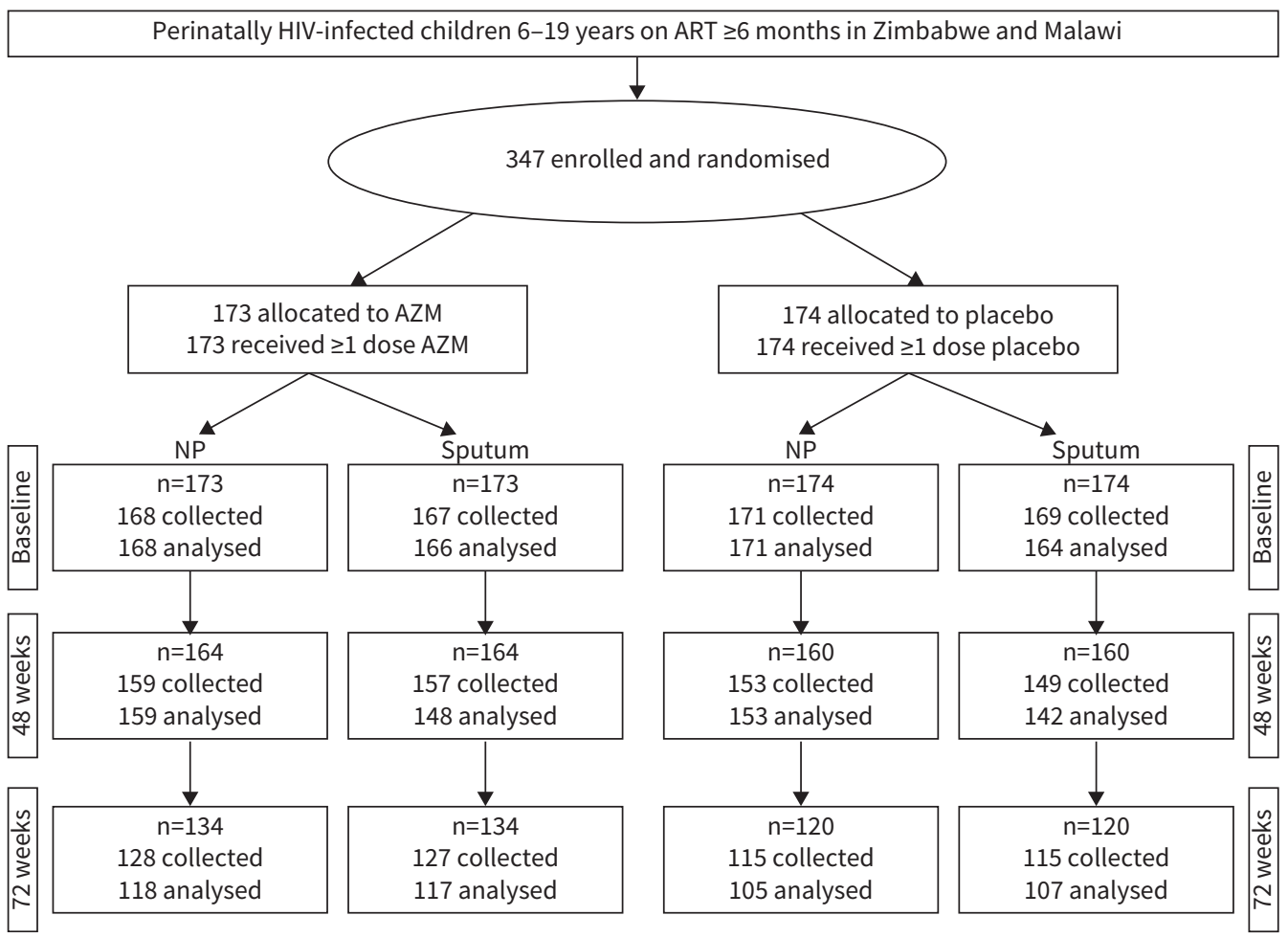

FIGURE 1 Flowchart of number of samples collected at each visit. ART: antiretroviral therapy; AZM: azithromycin; NP: nasopharyngeal. 
out of 347)) (table 1). NP and sputum samples were obtained from about $97 \%$ of all participants at baseline and 48 weeks and $86 \%$ at 72 weeks (figure 1).

Participants randomised to receive AZM were less likely to carry SP (aOR 0.2, 95\% CI 0.1-0.4, $\mathrm{p}<0.0001$ ), HI (aOR 0.3, 95\% CI 0.1-0.8, p=0.01) or MC (aOR 0.2, 95\% CI 0.1-0.8, p=0.02) (table 2) in their NP at 48 weeks than placebo. The carriage of any of the four species was lower in the AZM arm at 48 weeks (aOR 0.4, 95\% CI 0.2-0.7, p=0.001), but not at 72 weeks, compared to placebo (table 2). These observations were mirrored in the sputum samples (table 2).

At 48 weeks, the proportion of SP (62\% (18 out of 29) versus 13\% (8 out of 63 ), p<0.0001) or SA (91\% (31 out of 34 ) versus $3 \%$ (1 out of 31 ), $\mathrm{p}<0.0001$ ) isolates from the NP that were AZM-resistant was higher in the AZM arm than placebo (figures 2 and 3). However, at 72 weeks, there were no differences in the proportion of AZM-resistant strains, except for AZM-resistant SA (45\% (9 out of 20) in the AZM arm versus $4 \%$ ( 1 out of 26 ) in the placebo arm, $\mathrm{p}<0.001$ ) (figure 3). Overall AZM resistance (resistance in any of the four bacterial species) was higher in the AZM arm at 48 weeks (30\% (45 out of 154) versus $7 \%$ (10 out of 146), $\mathrm{p}<0.0001$ ) and remained so at 72 weeks (17\% (17 out of 102 ) versus $7 \%$ (7 out of 90), $\mathrm{p}<0.0001$ ) relative to placebo. The above observations were mirrored in sputum samples (figures 2 and 3 ).

\begin{tabular}{|c|c|c|c|}
\hline Characteristics & Details & AZM arm & Placebo arm \\
\hline Subjects $n$ & & 173 & 174 \\
\hline \multicolumn{4}{|l|}{ Demographic characteristics } \\
\hline Age years & Median (IQR) & $14.7(12.6-16.8)$ & $15.8(13.0-18.1)$ \\
\hline Sex & Female, $\mathrm{n}(\%)$ & $80(46.2)$ & $90(51.7)$ \\
\hline Currently in school & n $(\%)^{\#}$ & $146(84.5)$ & 139 (79.9) \\
\hline \multirow[t]{2}{*}{ Site } & Zimbabwe, n (\%) & $120(69)$ & $121(70)$ \\
\hline & Malawi, n (\%) & $53(31)$ & $53(30)$ \\
\hline \multicolumn{4}{|l|}{ HIV characteristics } \\
\hline Age years at diagnosis years & Median (IQR) & $7.2(3.5-9.9)$ & $8.3(5.2-11.1)$ \\
\hline Duration on ART years & Median (IQR) & $5.9(3.8-9.0)$ & $6.4(3.9-8.2)$ \\
\hline Age at ART initiation years & Median (IQR) & $8.2(5.0-11.2)$ & $8.9(6.7-11.6)$ \\
\hline HIV viral load log 10 copies $\cdot \mathrm{mL}^{-1}$ & Median (IQR) & $2.5(1.6-4.0)$ & $2.7(1.7-4.1)$ \\
\hline HIV viral load suppression & $<1000$ copies $\cdot \mathrm{mL}^{-1}, \mathrm{n}(\%)$ & $102(59.0)$ & $94(54.0)$ \\
\hline CD4 cell count $/ \mathrm{mm}^{3}$ & Median (IQR) & $601(417-784)$ & $550(325-779)$ \\
\hline \multirow[t]{2}{*}{ CD4 count categories } & $200+$ cells $/ \mathrm{mm}^{3}, \mathrm{n}(\%)$ & $157(91)$ & $156(89.7)$ \\
\hline & $<200$ cells $/ \mathrm{mm}^{3}, \mathrm{n}(\%)$ & $16(9)$ & $18(10.3)$ \\
\hline \multirow{2}{*}{ Antiretroviral regimen } & NNRTI, n (\%) & $127(73)$ & $131(75)$ \\
\hline & $\mathrm{PI}, \mathrm{n}(\%)^{\#}$ & $46(27)$ & $42(25)$ \\
\hline \multicolumn{4}{|l|}{ Lung function } \\
\hline $\mathrm{FEV}_{1} z$-score & Median (IQR) & $-1.94(-2.5--1.4)$ & $-2.0(-2.4--1.5)$ \\
\hline \multicolumn{4}{|l|}{ Clinical characteristics } \\
\hline Weight-for-age $z$-score & Median (IQR) & $-2.2(-3.0--1.4)$ & $-1.9(-2.8--1.2)$ \\
\hline Underweight & $\mathrm{n}(\%)^{4}$ & $98(56.6)$ & $83(47.7)$ \\
\hline Height-for-age $z$-score & Median (IQR) & $-2.1(-3.0--1.4)$ & $-1.9(-2.6--1.3)$ \\
\hline Stunted & $\mathrm{n}(\%)^{4}$ & $95(54.9)$ & $80(46.0)$ \\
\hline History of TB & n $(\%)^{\#}$ & $58(33.57)$ & $39(22.4)$ \\
\hline Admitted for chest problems in last year & n (\%) & $3(1.7)$ & $3(1.7)$ \\
\hline Current cough & $\mathrm{n}(\%)^{\#}$ & $13(7.5)$ & $18(10.3)$ \\
\hline Coughing up sputum & $\mathrm{n}(\mathrm{N})^{+}$ & $7(43)$ & $17(46)$ \\
\hline \multirow[t]{5}{*}{ MRC Dyspnoea Score } & $1, \mathrm{n}(\%)$ & $89(51)$ & $96(55)$ \\
\hline & $2, \mathrm{n}(\%)$ & $64(37)$ & $62(36)$ \\
\hline & $3, \mathrm{n}(\%)$ & $12(7)$ & $11(6.3)$ \\
\hline & $4, \mathrm{n}(\%)$ & $7(4)$ & $4(2.3)$ \\
\hline & $5, \mathrm{n}(\%)$ & $1(1)$ & $1(1)$ \\
\hline Cotrimoxazole prophylaxis & $\mathrm{n}(\%)$ & $157(92)^{\#}$ & $156(90)$ \\
\hline
\end{tabular}

AZM: azithromycin; IQR: interquartile range; NNRTI: nonnucleoside reverse transcriptase inhibitor; PI: protease inhibitor; $\mathrm{FEV}_{1}$ : forced expiratory volume in $1 \mathrm{~s}$, participants with missing responses are excluded from that variable; TB: tuberculosis; MRC: Medical Research Council. "Missing value: Currently attending school - $n=1$ AZM arm; Antiretroviral regimen, $n=1$, Placebo arm; History of TB - $n=1$, AZM arm; Current cough - $n=1$, AZM arm; Cotrimoxazole prophylaxis - $n=2, A Z M$ arm; ${ }^{~}$ : $z$-score $<-2 ;^{+}$: only asked for those with current cough. 
TABLE 2 Nasopharyngeal and sputum bacterial carriage at baseline, 48 weeks and 72 weeks in participants

\begin{tabular}{|c|c|c|c|c|c|c|c|c|c|c|}
\hline & \multicolumn{2}{|c|}{ Baseline } & \multicolumn{4}{|c|}{48 weeks } & \multicolumn{4}{|c|}{72 weeks } \\
\hline & AZM & Placebo & AZM & Placebo & aOR $(95 \% \mathrm{Cl})$ & $\mathrm{p}$-values & AZM & Placebo & aOR $(95 \% \mathrm{Cl})$ & $\mathrm{p}$-values \\
\hline \multicolumn{11}{|l|}{ Nasopharyngeal swabs } \\
\hline Subjects $\mathrm{n}$ & $168^{\#}$ & $171^{\#}$ & $159^{4}$ & $153^{4}$ & & & $118^{+}$ & $105^{+}$ & & \\
\hline Streptococcus pneumoniae & $74(44)$ & $83(49)$ & $29(18)$ & $64(41)$ & $0.2(0.1-0.4)$ & $<0.0001$ & $41(35)$ & $38(36)$ & $0.9(0.4-1.7)$ & 0.681 \\
\hline Staphylococcus aureus & $45(27)$ & $36(21)$ & $34(21)$ & $31(20)$ & $1.1(0.5-2.2)$ & 0.882 & $23(19)$ & $26(25)$ & $0.7(0.3-1.7)$ & 0.433 \\
\hline Haemophilus influenzae & $21(13)$ & $18(11)$ & $11(7)$ & $24(16)$ & $0.3(0.1-0.8)$ & 0.011 & $13(11)$ & $10(10)$ & $1.0(0.4-2.8)$ & 0.964 \\
\hline Moraxella catarrhalis & $26(15)$ & $21(12)$ & $7(4)$ & $17(11)$ & $0.3(0.1-0.8)$ & 0.015 & $9(8)$ & $7(7)$ & $0.9(0.3-2.9)$ & 0.848 \\
\hline Bacterial carriage (any) & $107(64)$ & $112(68)$ & $63(43)$ & $86(60)$ & $0.4(0.2-0.7)$ & 0.001 & $61(53)$ & $63(60)$ & $0.6(0.3-1.2)$ & 0.135 \\
\hline \multicolumn{11}{|l|}{ Sputum } \\
\hline Subjects $n$ & $166^{\S}$ & $164^{\S}$ & $148^{f}$ & $143^{f}$ & & & $116^{\# \#}$ & $106^{\# \#}$ & & \\
\hline Streptococcus pneumoniae & $43(26)$ & $38(23)$ & $17(11)$ & $34(24)$ & $0.4(0.2-0.8)$ & 0.008 & $20(17)$ & $26(25)$ & $0.6(0.3-1.2)$ & 0.122 \\
\hline Staphylococcus aureus & $51(31)$ & $46(28)$ & $46(31)$ & $37(26)$ & $1.4(0.7-2.8)$ & 0.323 & $40(34)$ & $37(34)$ & $1.1(0.5-2.3)$ & 0.788 \\
\hline Haemophilus influenzae & $4(2)$ & $7(4)$ & $3(2)$ & $19(13)$ & $0.1(0.0-0.4)$ & 0.002 & $6(5)$ & $9(8)$ & $0.5(0.1-1.6)$ & 0.221 \\
\hline Moraxella catarrhalis & $15(9)$ & $15(9)$ & $4(3)$ & $15(10)$ & $0.2(0.1-0.7)$ & 0.008 & $7(6)$ & $7(7)$ & $0.9(0.3-2.8)$ & 0.882 \\
\hline Bacterial carriage (any) & $82(49)$ & $79(48)$ & $62(42)$ & $77(54)$ & $0.6(0.3-1.0)$ & 0.056 & $56(48)$ & $60(57)$ & $0.8(0.4-1.4)$ & 0.395 \\
\hline
\end{tabular}

Data expressed as $n(\%)$ unless otherwise indicated. AZM: azithromycin; aOR: adjusted odds ratio. \#: samples not collected from five and three participants in AZM and placebo group at baseline respectively; ": samples not collected from five and seven participants in AZM and placebo group at 48 weeks respectively; ${ }^{+}$: samples not collected from 16 and 15 participants in AZM and placebo group at 72 weeks respectively; ${ }^{\S}$ : sputum samples were not collected from seven and 10 participants in AZM and placebo group at baseline respectively; ${ }^{f}$ : sputum samples not collected from 16 and 17 participants in AZM and placebo group at 48 weeks respectively; \#\#: sputum samples not collected from 18 and 14 participants in AZM and placebo group at 72 weeks respectively. 
NP
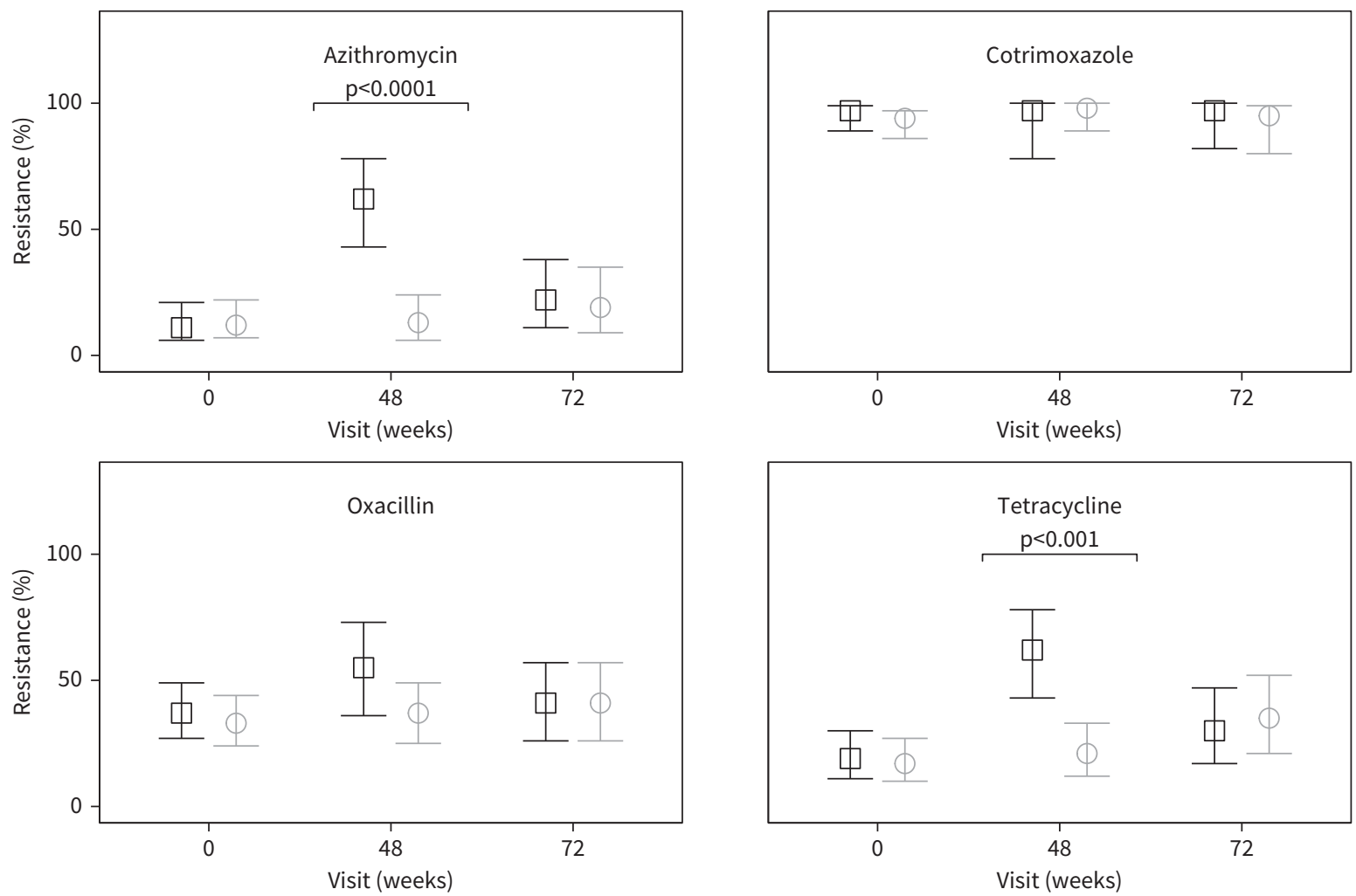

Trial-arm

$\boxminus A Z M$

$\odot$ Placebo

Sputum
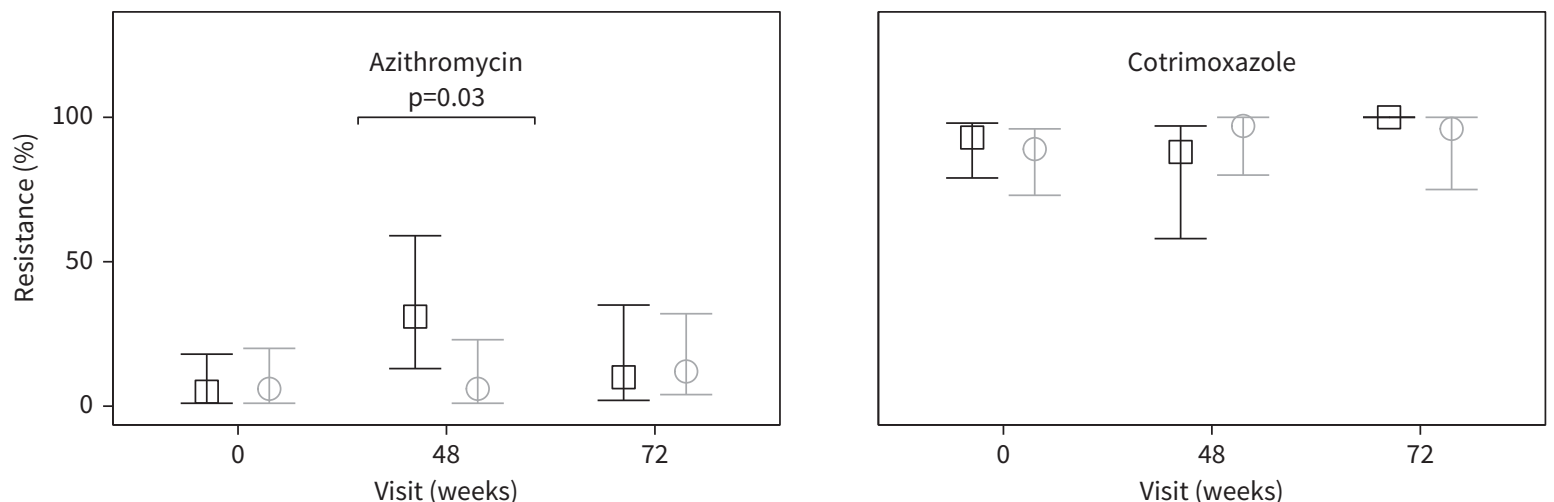

Trial-arm

isit (weeks)

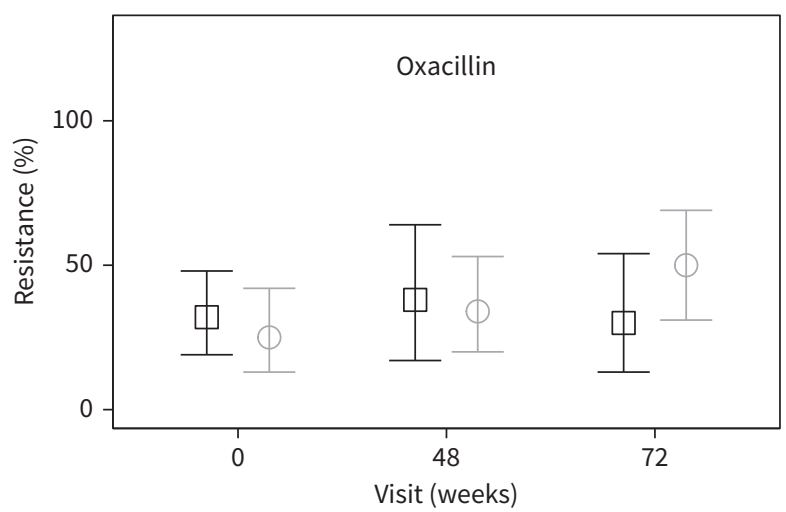

$\boxminus$ AZM

$\bigcirc$ Placebo

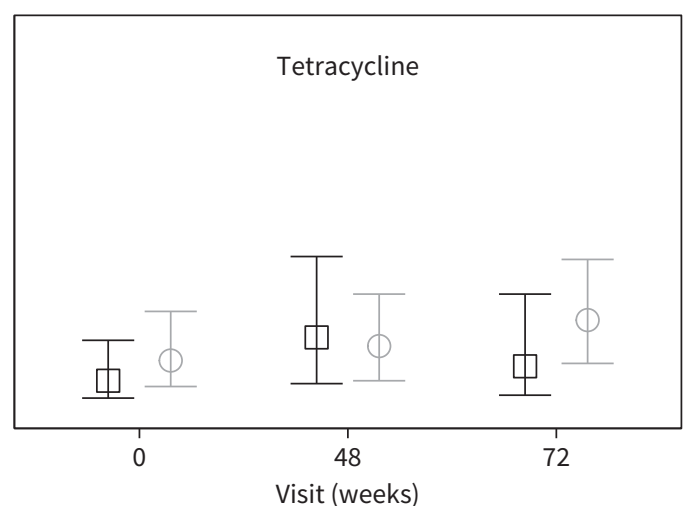

FIGURE 2 Antibiotic resistance profiles of Streptococcus pneumoniae isolated from the nasopharyngeal (NP) and sputum samples at baseline (0 week), 48 weeks and 72 weeks. The $x$ - and $y$-axes are sampling timepoints and percentage resistance, respectively. Number of isolates from NP samples at each timepoint for azithromycin (AZM) versus placebo were: baseline (71 versus 81), 48 weeks (29 versus 63 ), 72 weeks (37 versus 37 ). In the sputum, baseline (41 versus 36), 48 weeks (16 versus 32), 72 weeks (20 versus 26$)$. Oxacillin used as a surrogate for penicillin. 

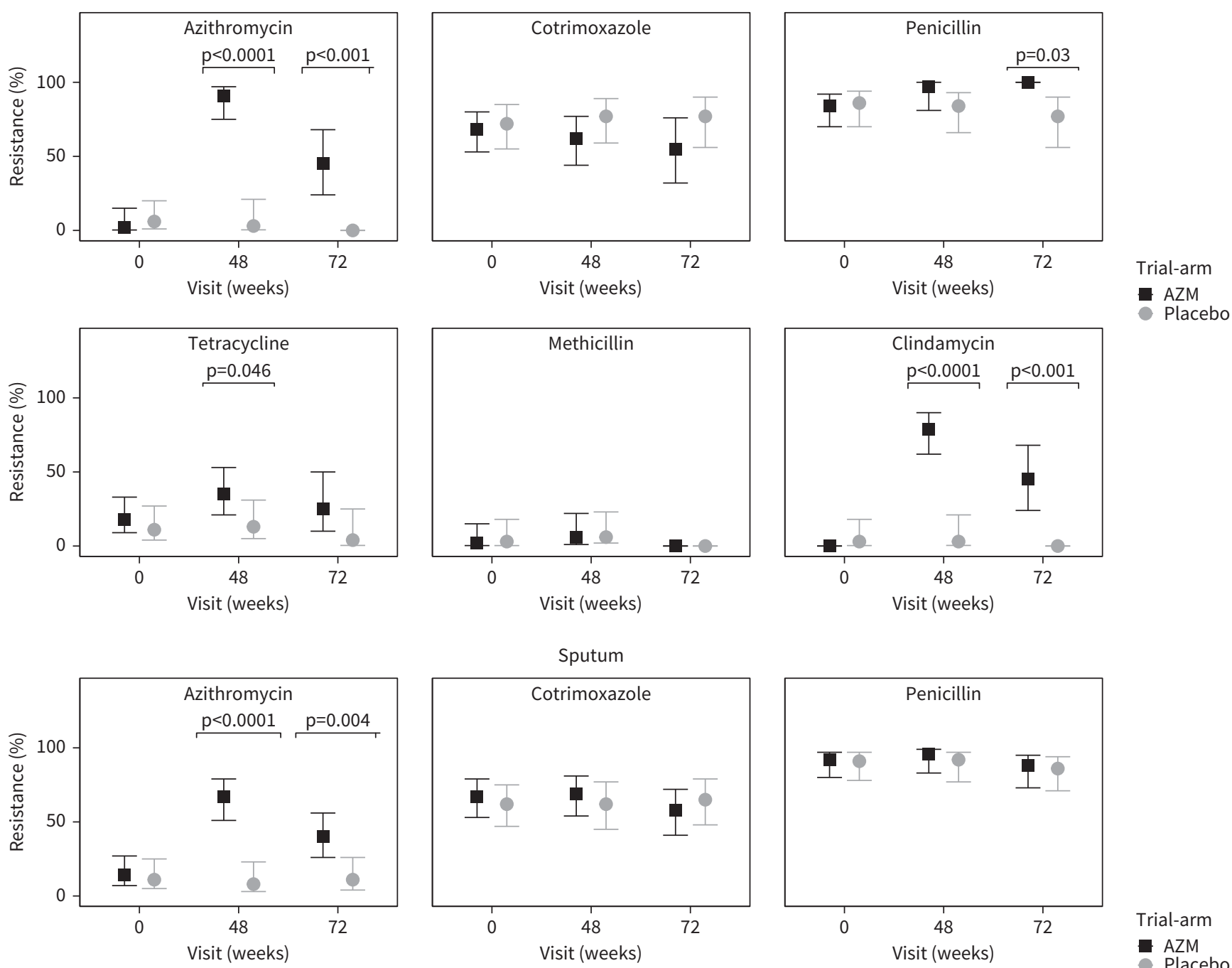

Sputum
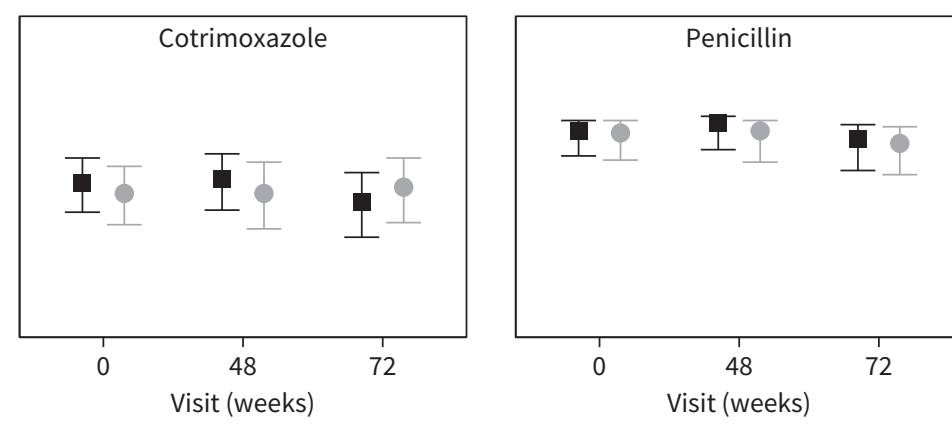

Trial-arm
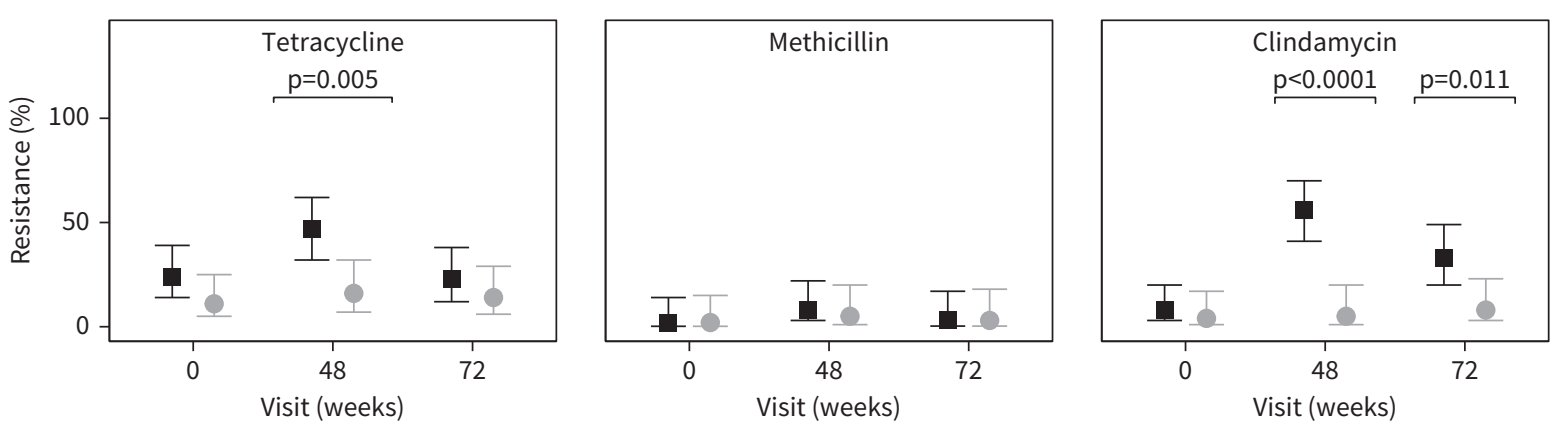

Placebo

FIGURE 3 Antibiotic resistance profiles of Staphylococcus aureus isolated from the nasopharyngeal and sputum samples at baseline (0 week), 48 weeks and 72 weeks. The $x$ - and $y$-axes are sampling timepoints and percentage resistance, respectively. Number of isolates from nasopharyngeal (NP) samples at each timepoint for azithromycin (AZM) versus placebo were: baseline (44 versus 36 ), 48 weeks (34 versus 31 ), 72 weeks (20 versus 26 ). In the sputum, baseline (49 versus 45 ), 48 weeks ( 45 versus 37 ), 72 weeks (40 versus 37 ).

The proportion of SP and SA in the NP that were tetracycline-resistant was also higher at 48 weeks in the AZM arm than placebo (figures 2 and 3, supplementary table S1). Clindamycin resistance in SA was higher in the AZM arm versus placebo at 48 weeks (79\% (27 out of 34 ) versus 3\% (1 out of 31), $\mathrm{p}<0.0001$ ) and remained higher at 72 weeks in the AZM arm compared to placebo (45\% (9 out of 20) versus $0 \%$ (0 out of 26 ), $\mathrm{p}<0.0001$ ) (figure 3, supplementary table S1). Carriage of methicillin-resistant SA 
remained low (6\%) throughout the study in both trial arms (figure 3, supplementary table S1). Penicillin (oxacillin) resistance in SP was moderate and there was no difference between trial arms; however, penicillin-resistant SA was higher in AZM than placebo at 72 weeks (supplementary table S1). Cotrimoxazole resistance levels in SA remained high ( 69\%), with no clear changes throughout the study period (supplementary table S1). There was no difference between the trial arms with HI and MC susceptibility to AZM, tetracycline, amoxicillin-clavulanate and cotrimoxazole (supplementary table S1, Figure S1-S2). There was a universal HI susceptibility to cefuroxime and ampicillin (supplementary table S1, Figure S1). The above observations were mirrored in sputum samples (figure 3, supplementary table S2, Figures S1-S2) except for tetracycline-resistant SP, which did not differ between trial arms at 48 weeks. Also, unlike in the NP, penicillin resistance in sputum SA was similar between trial arms at all visits (figure 3, supplementary table S2).

Adherence to AZM was associated with reduced likelihood of sputum SA and overall bacterial carriage at 48 weeks (supplementary table S3). Adherence was not associated with the carriage of resistant bacteria (supplementary tables S4 and S5). The main factors associated with SP carriage were male sex (NP only) and placebo allocation (NP and sputum) at 48 weeks and age at 72 weeks (supplementary table S6). Similarly, factors associated with NP and sputum SA carriage were non-adherence to AZM (NP and sputum, supplementary table S7), viral load suppression (sputum) and season of sampling (supplementary table S7). Placebo allocation and non-adherence to AZM was associated with increased likelihood of AZM-resistant SP and SA carriage in the NP or sputum (supplementary tables S8 and S9).

\section{Discussion}

Our study shows that HIV-infected children with CLD established on ART and long-term cotrimoxazole prophylaxis, who received 48 weeks of AZM therapy, were significantly less likely to carry SP, HI and $\mathrm{MC}$ in their NP or sputum compared to placebo at the end of treatment. However, these participants were more likely to carry AZM and tetracycline-resistant SP and SA at 48 weeks. AZM-, clindamycin- and tetracycline-resistant SA remained more prevalent in participants in the AZM arm at 72 weeks.

Our finding of reduced NP and sputum carriage of SP, HI and MC in response to long-term AZM therapy is consistent with previous studies [2, 6]. Similarly, the lack of an effect of long-term AZM therapy on SA carriage observed in our study was also reported in studies conducted in children with chronic suppurative lung disease in Australia and New Zealand [2], and cystic fibrosis in the Netherlands [14] and USA [3]. The reduction in SP, HI and MC prevalence in our study may be related to the almost universal susceptibility of these isolates to AZM at baseline or the consequence of improved alveolar macrophage phagocytic activity [15]. Phagocytosis is generally reduced in chronic lung conditions, especially COPD, but improves with AZM therapy [15]. A survey conducted from 2015 to 2018, the same time period as our study, reported a high SP carriage among children and adults in Malawi regardless of pneumococcal vaccination and HIV status [16]. Our finding that AZM reduced SP carriage may have important implications in reducing the risk of invasive SP disease as well as transmission. The lack of difference between trial arms in the carriage of SP, HI or MC at 72 weeks highlights that the antimicrobial effects of long-term AZM therapy may not be long-lasting. This is corroborated by reports from a randomised controlled trial of AZM among Indigenous Australian, Maori and Pacific Island children aged 1-8 years with chronic suppurative lung disease [2].

Long-term AZM therapy selected for AZM-resistant SP at the end of treatment. This is consistent with other studies that used AZM for both long- [1-3, 14, 17] and short-term [11, 18, 19] treatment periods. AZM resistance occurs soon after exposure, as evidenced by the recovery of resistant strains after a single AZM course or dose [11]. Previous studies revealed clonal expansion of existing resistant strains of SP following exposure to AZM [19]. Studies that involved mass AZM administration for trachoma also noted an increase in resistant SP, which resolved over time [20, 21]. Since the genetic changes that confer macrolide resistance may reduce the fitness of SP strains (their ability to compete with drug-susceptible strains), resistant SP strains were likely to have been displaced by susceptible strains once the antibiotic selective pressure was removed [2]. AZM use did not select for penicillin-resistant SP in our study. Although, we did not determine minimum inhibitory concentrations for the isolates, this non-selection of penicillin-resistant SP is consistent with previous studies that administered AZM for both short- [18] and long-term periods [2]. This is important as penicillins remain the first-line treatment for pneumococcal infections in sub-Saharan Africa.

Although the proportion of AZM-resistant SA declined at 72 weeks, AZM-resistant SA isolates remained significantly more common in the AZM arm at this visit. In a cystic fibrosis clinic in the Netherlands, the occurrence of AZM-resistant SA was common enough to warrant discontinuation of long-term AZM 
therapy among cystic fibrosis patients [1]. In contrast, in Denmark, a low prevalence of SA in cystic fibrosis patients at baseline with a further reduction during treatment with AZM made increased macrolide resistance in SA clinically insignificant [17]. It is possible that the baseline prevalence of SA may influence the effect of long-term AZM therapy on resistance. Additionally, the differences in strength and frequency of dosing of AZM across studies may also contribute to the variations in the observed patterns of resistance. SA is an important pathogen in HIV-infected patients where it causes bacteraemia leading to endocarditis [22]. Staphylococcal bacteraemia is about 17 times more likely to occur in a hospitalised HIV-infected adult than a HIV-negative patient [22]. The increase in AZM-resistant SA which persist beyond 6 months post-AZM in our setting is therefore concerning.

The increase in tetracycline (SP and SA) and clindamycin (SA only) resistance at 48 weeks is not surprising since mobile genetic elements that mediate resistance to macrolides co-carry genes that confer resistance to other antibiotics [23]. Of note, is the conjugative transposon Tn916-like element, which carries tet (tetracycline resistance) and erm genes [23]. The erm gene product modifies the bacterial ribosomal target site of macrolides, lincosamides (clindamycin) and streptogramin antibiotics, thereby conferring resistance. These transposons are found in both SP [23] and SA [24]; hence, the concurrent increase in resistance to macrolides, lincosamides and tetracyclines with AZM therapy in our study is expected [23]. These mobile genetic elements with multiple resistance genes can be shared between bacterial species and across genera, increasing the overall burden of drug resistance [25].

SP is the most common bacterial cause of community-acquired pneumonia globally [26]. Macrolideresistant SP is a major concern in the USA and other parts of the world as macrolides have been the first drug of choice in monotherapy for adults for decades [27]. There have been multiple reports of clinical failures resulting from macrolide-resistant SP when patients were treated with macrolides [28]. However, the clinical relevance of macrolide-resistant SP based on AST has remained controversial [28]. This is fuelled by observations that although macrolide-resistant SP, as determined by AST, has been on the rise, an increase in clinical failures due to these isolates has not been observed as frequently as expected (the so-called "in vitro in vivo" paradox) [27]. One explanation for this discrepancy is that the current in vitro resistance testing methods may not accurately model the action of an antibiotic in the body during an infection [26]. During inflammation, for instance, the level of AZM increases substantially because of intracellular accumulation within macrophages and leukocytes at the site of infection [26]. Furthermore, the potent immunomodulatory properties of macrolides, which influence the resolution of respiratory infections, are not accounted for by the AST method [26].

Although macrolides are not the first-line treatment for SP and SA respiratory infections in Malawi and Zimbabwe (penicillin and tetracyclines are used in Malawi and cotrimoxazole and amoxicillin in Zimbabwe), macrolides are indicated where there is treatment failure following amoxicillin use or in combination with ceftriaxone for severe illness (Malawi). They are also indicated in cases with penicillin allergy or atypical pneumonia in both countries. For SA infections (including those at other body sites, such as skin and soft tissue infections), cloxacillin is the first-line antibiotic; however, clindamycin is indicated in penicillin hypersensitivity. Therefore, the clinical relevance of AZM-resistant SP and SA and clindamycin-resistant SA among study participants and their contacts should be further investigated.

To address concerns about the transmission of AZM-resistant SA to untreated individuals, TRAMPER-STRANDERs et al. (2007) [29] compared the prevalence of AZM-resistant SA among cystic fibrosis patients on long-term AZM therapy and their household contacts. They found that although there was a higher AZM-resistant SA carriage among the cystic fibrosis patients (69.6\%), carriage among household contacts $(9.6 \%)$ was similar to the general population $(6.3 \%)$ of the Netherlands. Furthermore, the transmission of resistant isolates from the patients to their household contacts could not be proved by genotyping, except for one household. This finding suggests that although the potential risk of transmission of AZM-resistant SA exists, the probability is low. However, this observation may be quite different in an African household.

TAYLOR et al. [4, 30] showed that AZM's ability to reduce acute respiratory exacerbations is mediated by its capacity to reduce airway HI load, which is mirrored in our study. Our finding that AZM-resistant HI and MC strains were uncommon, and similar between trial arms at all visits, is consistent with previous clinical trials of long-term AZM therapy [2, 3]. However, other studies of long-term AZM observed an increase in AZM-resistant $\mathrm{HI}[1,31]$ and MC [3, 31]. The discordance across studies in the observed patterns of $\mathrm{HI}$ and MC resistance may be explained by the differences in the dose and duration of AZM used. Also, relatively few isolates of both bacterial species were identified in many studies (including our own) and may have been underpowered to detect resistance in these species. 
HuANG et al. [32] observed that acute respiratory exacerbations were associated with an increase in the relative abundance of the bacterial phylum Proteobacteria in patients with COPD. Antibiotic treatment reduced this relative abundance and was associated with resolution of symptoms. Broader changes in the airway microbiome, and not only in the specific bacterial species we measured, may modulate the effect of AZM in HIV-associated chronic lung disease. However, microbial dysbiosis may occur in other microbial niches, including the gut, following long-term AZM treatment, and the local and systemic consequences of such dysbiosis requires further study.

Given concerns around the emergence of resistance with long-term AZM therapy, the use of newer macrolide-based drugs that have potent immunomodulatory activity without resistance-promoting antibacterial effect should be considered. Hodge et al. [33] investigated two new macrolides with limited antibacterial activity against SP, SA, MC and HI. They found that these drugs improve macrophage phagocytosis of bacteria and therefore reduce bacterial carriage and associated acute exacerbations of CLD [33].

The strengths of our study are that it was a double-blinded, individually randomised, placebo-controlled, multicentre study among children with HIV-associated CLD in sub-Saharan African, a previously under-studied population. The study also analysed the persistence of the microbiological effect of long-term AZM therapy 6 months post-intervention. Furthermore, we also demonstrated that long-term AZM promoted resistance to other antibiotics (tetracycline and clindamycin). Study limitations include the lack of genomic investigation to delineate the mechanisms underlying antibiotic resistance and determine SP and HI serotypes. Furthermore, 27\% of participants' outcomes could not be assessed at 72 weeks due to withdrawal, death and loss to follow-up. However, our findings at this later time point are consistent with previous findings [2]. We could not determine when SA resistance to AZM, tetracycline and clindamycin resolved because our study concluded at 72 weeks from randomisation. Another limitation is that the study was not adequately powered to conclude whether AZM induced resistance in $\mathrm{HI}$ and MC as the number of isolates recovered from trial participants was low. We did not perform quantitative assessment of minimum inhibitory concentrations (MICs) of antibiotics, which may have identified increases in MICs that did not lead to changes in resistance category.

Long-term AZM therapy reduced the prevalence of SP, HI and MC in African children with HIV-associated CLD while increasing the carriage of AZM and tetracycline-resistant SP and SA at 48 weeks. Inducible clindamycin resistance also increased in SA at 48 weeks. However, these microbiological effects resolved 6 months post-intervention, except for AZM, tetracycline and clindamycin resistance in SA. Further studies are needed to determine whether resistance in SA persists beyond 72 weeks. The benefit of reduced acute exacerbations of HIV-associated CLD shown in this study [9] should be weighed against the largely temporary acquisition of antimicrobial resistance to AZM as well as other antibiotics.

Acknowledgements: We acknowledge the support of the staff at the Division of Medical Microbiology and the Dube lab, especially Gillian Ndhlovu at the University of Cape Town. The Global Health and Vaccination Research (GLOBVAC) Programme of the Medical Research Council of Norway funded this study. This work was also supported by the National Institute for Health Research (NIHR) Global Health Research Unit on Mucosal Pathogens using UK aid from the UK Government (Project number 16/136/46). RSH is a NIHR Senior Investigator. The views expressed are those of the authors and not necessarily those of the NIHR, the Department of Health and Social Care. MN is supported by an Australian National Health and Medical Research Council Investigator Grant [APP1174455]. REA is funded by the L'Oréal UNESCO For Women in Science Fellowship, Margaret McNamara Education Grants and the Swedish International Development Cooperation Agency (SIDA) through the Organisation of Women in Science for the developing world (OWSD) PhD Fellowship. FSD is supported by the National Research Foundation of South Africa (112160), Future Leaders - African Independent Research (FLAIR) Fellowship and the National Institute for Health Research (NIHR) Global Health Research Unit on Mucosal Pathogens (MPRU) using UK aid from the UK Government and the University of Cape Town. RAF is funded by the Wellcome Trust. AMR is supported by the UK Medical Research Council (MRC) and the UK Department for International Development (DFID) under the MRC/DFID Concordat agreement, which is also part of the EDCTP2 Programme supported by the European Union, Grant Ref: MR/R010161/1.

Provenance: Submitted article, peer reviewed.

ClinicalTrials.gov Identifier: NCT02426112

Data availability: All de-identified data can be accessed at dryad.org (https://doi.org/10.5061/dryad.5dv41ns71) and analysis codes are available at Zenodo.org (https://doi.org/10.5281/zenodo.5712334). 
Author contributions: MPN, FSD and RAF conceived the study. REA conducted laboratory experiments and wrote the first manuscript draft, supervised by MPN and FSD. CB assisted with laboratory experiments. Statistical analysis was conducted by REA, supported by AMR and VS. GM and LN coordinated the trial in Zimbabwe and Malawi, respectively. BW and RH reviewed the manuscript. JOO and RAF are trial PIs. All authors contributed to the manuscript, and all authors have read and approved the final version.

Conflict of interest: Regina E. Abotsi has nothing to disclose. Mark Nicol reports grants from Australian National Health and Medical Research Council, and Medical Research Council of Norway, paid to the institution, outside the submitted work. Grace McHugh has nothing to disclose. Victoria Simms has nothing to disclose. Andrea M Rehman reports support for the present manuscript from Norwegian Research Council, payment to institution. Grants of contracts received from UK Medical Research Council (MRC) and the UK Department for International Development (DFID) under the MRC/DFID Concordat agreement which is also part of the EDCTP2 programme supported by the European Union, and Bill and Melinda Gates Foundation, payments to institution, outside the submitted work. Payment or honoraria for lectures, presentations, speakers bureaus, manuscript writing or educational events from External examiner for Middlesex University MSc in Public Health, outside the submitted work. Unpaid Participant on DSMB for INTE-AFRICA study. Unpaid Chair of the Royal Statistical Society International Development Section Committee. Charmaine Barthus has nothing to disclose. Lucky G. Ngwira has nothing to disclose. Brenda Kwambana-Adams has nothing to disclose. Robert $S$ Heyderman has nothing to disclose. Jon $\varnothing$ Odland has nothing to disclose. Rashida Ferrand reports support for the present manuscript from the BREATHE Trial was funded by GLOBVAC initiation of the Norwegian Research Council, payment to institution. Grants or contracts from the Wellcome Trust through a Senior Fellowship in Clinical Science, paid to institution, outside the submitted work. Felix S. Dube has nothing to disclose.

Support statement: Global Health and Vaccination Research (GLOBVAC) Programme of the Medical Research Council of Norway.

\section{References}

1 Phaff SJ, Tiddens HAWM, Verbrugh HA, et al. Macrolide resistance of Staphylococcus aureus and Haemophilus species associated with long-term azithromycin use in cystic fibrosis. $J$ Antimicrob Chemother 2006; 57: 741-746.

2 Hare KM, Grimwood K, Chang AB, et al. Nasopharyngeal carriage and macrolide resistance in Indigenous children with bronchiectasis randomized to long-term azithromycin or placebo. Eur J Clin Microbiol Infect Dis 2015; 34: 2275-2285.

3 Albert RK, Connett J, Bailey WC, et al. Azithromycin for prevention of exacerbations of COPD. N Engl J Med 2011; 365: 689-698.

4 Taylor SL, Leong LEX, Mobegi FM, et al. Long-term azithromycin reduces Haemophilus influenzae and increases antibiotic resistance in severe asthma. Am J Respir Crit Care Med 2019; 200: 309-317.

5 Serisier DJ. Risks of population antimicrobial resistance associated with chronic macrolide use for inflammatory airway diseases. Lancet Respir Med 2013; 1: 262-274.

6 Li H, Liu D-H, Chen L-L, et al. Meta-analysis of the adverse effects of long-term azithromycin use in patients with chronic lung diseases. Antimicrob Agents Chemother 2014; 58: 511-517.

7 Brusselle GG, Vanderstichele C, Jordens $P$, et al. Azithromycin for prevention of exacerbations in severe asthma (AZISAST): a multicentre randomised double-blind placebo-controlled trial. Thorax 2013; 68: 322-329.

8 Ferrand RA, Desai SR, Hopkins C, et al. Chronic lung disease in adolescents with delayed diagnosis of vertically acquired HIV infection. Clin Infect Dis 2012; 55: 145-152.

9 Ferrand RA, McHugh G, Rehman AM, et al. Effect of once-weekly Azithromycin vs placebo in children with HIV-associated chronic lung disease: the BREATHE randomized clinical trial. JAMA Netw Open 2020; 3: e2028484.

10 Abotsi RE, Nicol MP, McHugh G, et al. Prevalence and antimicrobial resistance profiles of respiratory microbial flora in African children with HIV-associated chronic lung disease. BMC Infect Dis 2021; 21: 216.

11 Bojang E, Jafali J, Perreten V, et al. Short-term increase in prevalence of nasopharyngeal carriage of macrolide-resistant Staphylococcus aureus following mass drug administration with azithromycin for trachoma control. BMC Microbiol 2017; 17: 75.

12 CLSI. Performance Standards for Antimicrobial Susceptibility Testing. 28th Edn. Wayne, PA, Clinical Laboratory Standards Institute, 2018.

13 Twisk J, Bosman L, Hoekstra T, et al. Different ways to estimate treatment effects in randomised controlled trials. Contemp Clin Trials Commun 2018; 10: 80-85.

14 Tramper-Stranders GA, Wolfs TFW, Fleer A, et al. Maintenance azithromycin treatment in pediatric patients with cystic fibrosis: long-term outcomes related to macrolide resistance and pulmonary function. Pediatr Infect Dis J 2007; 26: 8-12. 
Hodge S, Reynolds PN. Low-dose azithromycin improves phagocytosis of bacteria by both alveolar and monocyte-derived macrophages in chronic obstructive pulmonary disease subjects. Respirol Carlton Vic 2012; 17: 802-807.

16 Swarthout TD, Fronterre C, Lourenço J, et al. High residual carriage of vaccine-serotype Streptococcus pneumoniae after introduction of pneumococcal conjugate vaccine in Malawi. Nat Commun 2020; 11: 2222.

17 Hansen CR, Pressler T, Hoiby N, et al. Long-term, low-dose azithromycin treatment reduces the incidence but increases macrolide resistance in Staphylococcus aureus in Danish CF patients. J Cyst Fibros 2009; 8: 58-62.

18 Skalet AH, Cevallos V, Ayele B, et al. Antibiotic selection pressure and macrolide resistance in Nasopharyngeal Streptococcus pneumoniae: a cluster-randomized clinical trial. PLoS Med 2010; 7: 12.

19 Keenan JD, Klugman KP, McGee L, et al. Evidence for clonal expansion after antibiotic selection pressure: pneumococcal multilocus sequence types before and after mass Azithromycin treatments. J Infect Dis 2015; 211: 988-994.

20 Leach AJ, Shelby-James TM, Mayo M, et al. A prospective study of the impact of community-based azithromycin treatment of trachoma on carriage and resistance of Streptococcus pneumoniae. Clin Infect Dis 1997; 24: 356-362.

21 Haug S, Lakew T, Habtemariam G, et al. The decline of pneumococcal resistance after cessation of mass antibiotic distributions for trachoma. Clin Infect Dis 2010; 51: 571-574.

22 Senthilkumar A, Kumar S, Sheagren JN. Increased incidence of Staphylococcus aureus bacteremia in hospitalized patients with acquired immunodeficiency syndrome. Clin Infect Dis 2001; 33: 1412-1416.

23 Chancey ST, Agrawal S, Schroeder MR, et al. Composite mobile genetic elements disseminating macrolide resistance in Streptococcus pneumoniae. Front Microbiol 2015; 6: 26.

24 De Vries LE, Christensen H, Skov RL, et al. Diversity of the tetracycline resistance gene tet (M) and identification of Tn 916- and Tn 5801-like (Tn 6014) transposons in Staphylococcus aureus from humans and animals. J Antimicrob Chemother 2009; 64: 3.

25 Schroeder MR, Stephens DS. Macrolide resistance in Streptococcus pneumoniae. Front Cell Infect Microbiol 2016; 6: 98.

26 Hoban DJ, Zhanel GG. Clinical implications of macrolide resistance in community-acquired respiratory tract infections. Expert Rev Anti Infect Ther 2006; 4: 973-980.

27 Nuermberger E, Bishai WR. The clinical significance of macrolide-resistant Streptococcus pneumoniae: it's all relative. Clin Infect Dis 2004; 38: 99-103.

28 Klugman KP, Lonks JR. Hidden epidemic of macrolide-resistant pneumococci. Emerg Infect Dis 2005; 11: 802-807.

29 Tramper-Stranders GA, van der Ent CK, Gerritsen SAM, et al. Macrolide-resistant Staphylococcus aureus colonization in cystic fibrosis patients: is there transmission to household contacts? J Antimicrob Chemother 2007; 60: 665-668.

30 Taylor SL, Ivey KL, Gibson PG, et al. Airway abundance of Haemophilus influenzae predicts response to azithromycin in adults with persistent uncontrolled asthma. Eur Respir J 2020; 56: 2000194.

31 Altenburg J, de Graaff CS, Stienstra Y, et al. Effect of azithromycin maintenance treatment on infectious exacerbations among patients with non-cystic fibrosis bronchiectasis: the BAT randomized controlled trial. JAMA 2013; 309: 1251-1259.

32 Huang YJ, Sethi S, Murphy T, et al. Airway microbiome dynamics in exacerbations of chronic obstructive pulmonary disease. J Clin Microbiol 2014; 52: 2813-2823.

33 Hodge S, Tran HB, Hamon R, et al. Nonantibiotic macrolides restore airway macrophage phagocytic function with potential anti-inflammatory effects in chronic lung diseases. Am J Physiol Lung Cell Mol Physiol 2017; 312: L678-L687. 